\title{
A ESTRATÉGIA DA SELEÇÃO NO ESPORTE COMPETITIVO: UM ESTUDO DESCRITIVO- EXPLORATÓRIO COM ATLETAS CORREDORES COM MAIS DE 45 ANOS
}

\author{
Sionara Tamanini de Almeida ${ }^{1}$ \\ Ricardo Pedrozo Saldanha ${ }^{2}$ \\ Carlos Adelar Abaide Balbinotti ${ }^{3}$ \\ Johannes Doll ${ }^{4}$
}

resumo

Baltes e Baltes (1990) propuseram um modelo de desenvolvimento adaptativo denominado de seleção, otimização e compensação (Modelo SOC). O artigo concentrou análise na estratégia da seleção e apresentou como objetivo analisar como atletas corredores selecionaram suas ações na prática esportiva. Foi realizada uma triagem com 83 atletas para, posteriormente, 11 atletas (média de idade

1 Licenciatura Plena em Educação Física pela Universidade Federal do Rio Grande do Sul (UFRGS). Fisioterapia pela Rede Metodista de Educação do Sul (IPA). Mestrado em Educação pela Universidade Federal do Rio Grande do Sul (UFRGS). Professora de Educação Física SMED. E-mail: sionara@cpovo.net 2 Licenciatura Plena em Educação Física pela Rede Metodista de Educação do Sul (IPA). Mestrado em Ciências do Movimento Humano pela Universidade Federal do Rio Grande do Sul (UFRGS). Professor da Faculdade Cenecista de Osório (FACOS). E-mail: ricardopsaldanha@yahoo.com.br

3 Licenciatura Plena em Educação Física pela Universidade Federal do Rio Grande do Sul (UFRGS). Doutor em Ciências do Movimento Humano pela Universidade Federal do Rio Grande do Sul (UFRGS). Professor da Universidade Federal do Rio Grande do Sul (UFRGS). E-mail: cbalbinotti@terra.com.br

4 Graduação em Teologia Católica pela Eberhard Karls Universität Tübingen. Graduação em Educação pela Erziehungswissenschaftliche Hochschule Landau. Doutorado em Educação pela Universität Koblenz Landau. Professor da Universidade Federal do Rio Grande do Sul (UFRGS). Email: johannes.doll@ufrgs.br 
59,91 \pm 0,58 anos) preencherem um questionário e realizarem uma entrevista semiestruturada. Os dados provenientes das entrevistas demonstraram que os atletas utilizaram a estratégia da seleção para direcionar sua vida ou superar perdas/dificuldades e com isso permanecerem na prática da corrida ou melhorarem a performance esportiva por mais tempo.

palavras - chave:

Envelhecimento. Educação Física. Psicologia do Desenvolvimento.

\section{Introdução}

O presente estudo situa-se na área da Pedagogia do Treinamento Desportivo, mais especificamente na perspectiva da orientação pedagógica para o desenvolvimento da performance desportiva de atletas corredores (corridas de velocidade ou fundistas) da categoria Masters - acima de 45 anos - do sexo masculino. A base teórica utilizada para esta pesquisa foi a perspectiva life-span - psicologia do desenvolvimento e envelhecimento - quando analisada de uma forma mais geral, e o metamodelo de seleção, otimização e compensação mais especificamente que operacionaliza a crença da life-span no âmbito do envelhecimento bem sucedido (LI; FREUND, 2005; BALTES, 1987). O processo de desenvolvimento humano é um fenômeno complexo que se estende ao longo da vida de forma multidirecional: variabilidade interindividual, intraindividual, e multidimensional. Essas diferentes direções no desenvolvimento referem-se ao potencial individual de mudança, flexibilidade e resistência para lidar com desafios e exigências (plasticidade) (BALTES; STAUDINGER; LINDENBERGER, 1999).

O processo de desenvolvimento/envelhecimento apresenta uma expressão conjunta de características de crescimento (ganhos) e declínios (perdas). Nenhuma fase do desenvolvimento é somente contemplada por ganhos ou apresenta somente perdas. Ambas estão em constante mudança em uma balança que deverá ser equilibrada através de capacidades adaptativas que o sujeito possui (BALTES, 1987).

Baltes e Baltes (1990) desenvolveram um modelo, denominado de Modelo SOC - Seleção, Otimização, e Compensação - que fornece uma estrutura geral 
para a compreensão das mudanças comportamentais e de resiliência ${ }^{5}$ durante a vida. O modelo elabora a pressuposição de que durante a vida, as pessoas encontram certas oportunidades (exemplo, educação), bem como limitações de recursos (exemplo, doenças) que podem ser manejadas de forma adaptativa através da combinação de três componentes: seleção, otimização e compensação. Tais estratégias podem ser vislumbradas no dia a dia das pessoas, em que cada uma corresponde a formas de adaptação na escolha de metas e ações para concretizá-las.

A estratégia da seleção refere-se à necessidade da escolha de metas e ações, em razão de que não podemos buscar e/ou alcançar todas as oportunidades que nos aparecem (seleção eletiva). Além disso, quando se processa alguma perda precisamos concentrar nossa atenção em ações específicas para buscarmos novas metas, ou adaptar-nos a novos padrões (seleção baseada em perdas) (ROHR; LANG, 2009; FREUND; 2002; FREUND, BALTES, 1998; BAJOR; BALTES, 2003; LI; FREUND, 2005). A estratégia da otimização, segundo os autores, é um processo no qual o indivíduo adquire, aperfeiçoa e aplica recursos para atingir seus objetivos. São ações que procuram maximizar ganhos em determinada área. E, por fim, a estratégia da compensação designa a utilização de algum recurso para a manutenção de determinado nível de funcionamento desejável, quando alguma perda se instala no indivíduo (ROHR; LANG, 2009; YEUNG; FUNG, 2009; JOPP; SMITH, 2006)

$\mathrm{O}$ artigo que segue, concentra sua atenção na estratégia da seleção, a fim de analisar como os atletas, praticantes de corrida, que participam periodicamente de competições e com mais de 45 anos, selecionaram suas ações para continuarem no esporte, mesmo com a presença de dificuldades, ou perdas relativas ao processo de envelhecimento. A seleção de ações é uma das formas de adaptação a determinadas circunstâncias que se apresentam na vida, defendida por Baltes e Baltes (1990).

Sendo assim, algumas questões norteadoras foram determinantes para investigar como esses atletas estão construindo e/ou vivenciando o processo de envelhecimento. Como os atletas conseguem permanecer na atividade esportiva competitiva por tantos anos? Quais suas motivações? O que os faz melhorar a performance? Há alguma perda? Caso percebam perdas, como eles

\footnotetext{
5 O termo resiliência de acordo com Staudinger, Marsiske e Baltes (1995) diz respeito a dois tipos de respostas adaptativas. A primeira se refere à manutenção do desenvolvimento normal, apesar das ameaças e riscos presentes na vida das pessoas. E, a segunda é relativa à recuperação após um trauma. A resiliência é mutável de acordo com as circunstâncias e desafios. Pode ser considerada como um tipo de plasticidade, quando incluída em um amplo espectro de possíveis modificações na capacidade adaptativa, pois diz respeito ao potencial para a manutenção e a recuperação dos níveis de adaptação normal.
} 
se ajustam, uma vez que continuam na atividade? Há algum ganho apesar da idade? Como lidam com suas lesões?

Tais questões foram traçadas a partir da análise do ambiente de pesquisa associado ao que foi encontrado, ou não na literatura científica sobre o tema. Durante a análise realizada na literatura científica, constatou-se pouco investimento em pesquisas com os atletas masters. O que se dispõe são artigos que tratam da questão fisiológica do seu envelhecimento (ALLISON; SEALS, 2011; FAULKNER et al, 2008; MORLEY, 2000; MAHARAM et al, 1999), outros se preocupam com as lesões (CHEN; MEAR; HAWKINGS, 2005) ou com a reabilitação dos atletas (BROWN, 1989). Além disso, há artigos preocupados com a questão do atleta idoso (BENTO, 1999; CAMPBELL; GEIK, 2004). Com relação ao número de atletas master existente, o que é encontrado são estatísticas incertas e confusas com relação aos atletas mais velhos que misturam as diferentes modalidades esportivas para os atletas com mais de 35 anos de idade (SEGAL; CRESPO; SMIT, 1998).

Por fim, quando se analisou o modelo SOC descobriu-se que existe uma carência de estudos que relacionem o modelo aos atletas masters. Apenas foi encontrada a indicação de um exemplo de como acontece o modelo em maratonistas, no texto sobre velhice bem sucedida, de Baltes e Carstensen (2000).

\section{Metodologia da pesquisa}

\section{Amostra}

Na primeira fase desta pesquisa foram avaliados 83 atletas corredores (corridas de velocidade ou fundistas) do sexo masculino participantes de competições esportivas, com idades variando entre 45 e 78 anos $(\bar{\chi}=54,22$; $\mathrm{DP}= \pm 8,17$ anos). Os atletas eram provenientes de diferentes localidades do Brasil (Paraná, Bahia, Rio Grande do Sul e São Paulo). Para a segunda fase da pesquisa, foram entrevistados 11 atletas ( $\bar{\chi}=59,91$; $\mathrm{DP}= \pm 10,58$ anos) dos 83 avaliados anteriormente.

\section{Instrumentos de Coleta}

Para esta pesquisa foram utilizados três instrumentos: um contendo informações sócio-demográficas (dados gerais do atleta; sobre treinamento; 
informações sobre saúde); o Inventário SOC; e uma entrevista semiestruturada. O inventário trata-se de uma ficha composta de 12 itens que avaliam as estratégias de gerenciamento de vida propostas por Baltes e Baltes (1990) - seleção eletiva, seleção baseada em perdas, otimização e compensação - o modelo SOC. Quando no preenchimento, a resposta foi indicada através da escolha entre duas frases que demonstravam atitudes do dia a dia do indivíduo. Dessa forma, o atleta deveria escolher aquela que mais se aproximava do seu modo de agir. A entrevista semiestruturada foi aplicada com uma estrutura prévia composta por 21 questões.

\section{Procedimentos da Pesquisa}

Para a realização da pesquisa foram tomados todos os cuidados éticos (Termos de Consentimento Livre e Esclarecido), sugeridos pela Universidade Federal do Rio Grande do Sul baseados na Resolução no 196, de 10 de outubro de 1996, no qual definem-se diretrizes e normas regulamentadoras de pesquisas envolvendo seres humanos.

O processo de pesquisa foi iniciado com a realização de um estudo piloto com a finalidade de conhecer e explorar o campo de estudo. A partir disso, o processo de pesquisa apresentou duas fases distintas. A primeira foi uma triagem, no qual os atletas foram convidados a preencherem uma ficha que continha o Inventário SOC na sua versão reduzida (FREUND; BALTES, 2002).

Após a primeira fase, os sujeitos que obtiveram o score igual ou superior a 9 pontos nesse inventário ${ }^{6}$, foram convidados através de um telefonema, ou via correio eletrônico a preencherem o questionário sócio-demográfico (RICHARDSON, 1999) e a realizarem a entrevista semiestruturada (GIL, 1987; GASKELL, 2003).

\section{Apresentação e Discussão dos Resultados}

Apoiados nas informações recolhidas no questionário sócio-demográfico, constatamos que os atletas participantes da segunda fase da pesquisa iniciaram a prática esportiva, em média, aos 35 anos de idade. Todos participavam de competições e treinavam regularmente com frequência média de 4 vezes por semana, em torno de $45 \mathrm{Km}$ totais de treinamento semanal. A maioria dos atletas (9) era vinculada a algum clube ou associação de corredores. Com relação às atividades profissionais, o grupo entrevistado apresentou 6 sujeitos 
aposentados e 5 não aposentados. No entanto, 4 atletas não exercem nenhuma atividade profissional e 7 continuam ativos em alguma profissão/ocupação.

Os dados que seguem foram discutidos a partir das temáticas que se apresentaram e que se caracterizaram nas estratégias do modelo SOC utilizadas. O presente estudo mostra-se inédito pelo reduzido número de investigações que aprofundam a análise no modelo de seletiva otimização com compensação no Brasil e com uma amostra de atletas master. Foi encontrada apenas uma investigação semelhante realizada por Lichtenfels (2007) em grupo de idosos distinto do presente estudo, ou seja, a autora realizou uma pesquisa com 30 idosos (idades entre 62 e 82 anos), moradores do Morro da Cruz e da Ilha da Pintada, no município de Porto Alegre - RS, com a finalidade de descrever e identificar as maneiras e as capacidades de adaptação, de enfrentamento e de atuação do adulto idoso em seu cotidiano. A pesquisa teve metodologia qualitativa (abordagem fenomenológica) e levantou o uso das estratégias SOC pelos idosos, revelando a importância das escolhas de ações realizadas, apesar das muitas restrições causadas pela pobreza em que viviam em termos socioeconômicos e culturais.

O construto teórico que o metamodelo propõe é de acordo com os autores Freund e Baltes (2002), diferenciado e conceitualmente único, o que o torna importante de ser aplicado em outros contextos.

Selecionar ações significa escolher qual caminho seguir ou que ação se opta. No entanto, conceitualmente podemos diferenciá-la a partir do contexto em que a estratégia é desenvolvida, ou seja, a seleção é subdividida em seleção eletiva e seleção baseada em perdas, onde o limite entre uma e outra estratégia acontece a partir da existência ou não de uma perda que pode se processar na vida do atleta (ROHR; LANG, 2009; BALTES; CARTENSEN, 1999 e 2000; BALTES, STAUDINGER; LINDENBERGER, 1999; FREUND; BALTES, 2002, 2002b).

Durante a entrevista, a estratégia da seleção foi utilizada e demonstrou que em determinado momento da vida o atleta direcionou a atenção para objetivos específicos no esporte, ou em sua vida dentre uma variedade de possíveis possibilidades que poderia se engajar (ROHR; LANG, 2009; JOPP; SMITH, 2006; FREUND; BALTES, 2002b). Com a estratégia, houve a garantia de canalizar os potenciais existentes em direção ao crescimento no processo de desenvolvimento.

Durante a escolha do próprio esporte, a estratégia foi utilizada de forma eletiva na época da juventude ou em anos mais atuais dentre outros esportes que poderiam escolher. A seleção aconteceu em função de restrições ambien- 
tais relativas ao trabalho, características pessoais percebidas ou do próprio esporte. Exemplificamos através do seguinte depoimento:

"Quando jovem eu podia jogar futebol, eu jogava muito bem, mas eu escolhi a
corrida porque eu gosto de correr. Eu gosto de correr, me sinto bem. Futebol
eu jogo bem, mas não gosto (A4 - 57 anos)".

A estratégia da seleção (eletiva) também foi apontada como uma ação mais atual, utilizada no momento da prova. Ela se mostrou na presença de algumas atitudes preparatórias comuns para o momento da competição. O atleta 1 exemplifica demonstrando sua escolha e seu compromisso com relação ao esporte, conforme fala:

"Não tomo bebida alcoólica na semana de prova... Não cometo excessos como ir a festas, dormir tarde, bebidas... Com o passar dos anos creio que tenho melhorado [performance], uma vez que reduzi sensivelmente as noitadas [...] (A1 - 52 anos)".

O consumo de álcool associado ao esporte existiu, existe e, por mais que medidas sejam tomadas, sempre existirá. O mais importante é que as pessoas estejam sempre informadas quanto a seus efeitos, riscos e benefícios, para que possam optar em consumir, ou não, esse tipo de bebida (SANTOS; TINUCCI, 2004). Para isso, o Colégio Americano de Medicina do Esporte - ACSM (2010) se posicionou oficialmente com relação ao uso do álcool, pontuando o que a literatura mostra sobre a utilização da bebida pelo esportista.

Como efeito agudo da administração do álcool pode exercer um efeito deletério sobre uma ampla variedade de habilidades psicomotoras, tais como tempo de reação, coordenação motora ampla e específica olho-mão, precisão dos movimentos e equilíbrio. Também pode haver uma redução da força muscular, da potência e endurance muscular localizada, da velocidade e endurance cardiovascular e, portanto, pode ser causa de decréscimo nos níveis de performance. A ingestão aguda de álcool parece não influenciar substancialmente as funções metabólicas, ou fisiológicas essenciais para o desempenho físico (metabolismo energético, consumo máximo de oxigênio, freqüência cardíaca, volume sistólico, débito cardíaco, fluxo sanguíneo muscular, diferença arteriovenosa de oxigênio e na dinâmica respiratória), mas pode impedir a regulação da temperatura corporal durante o exercício prolongado em ambientes frios. Além disso, o consumo exagerado de álcool pode produzir em longo prazo alterações patológicas no fígado, coração, cérebro e músculo que pode levar à incapacidade e morte (ACMS, 2010). 
Apesar do posicionamento da ACSM, no artigo de Santos e Tinucci (2004), há o indicativo de benefícios que o consumo de até duas doses diárias de álcool pode trazer para o atleta, assim como para qualquer indivíduo, como o aumento da sensação de bem-estar e a redução de risco cardiovascular.

Com relação à estratégia de seleção baseada em perdas, ela adapta o nível de expectativa do sujeito a perdas para restabelecer uma nova hierarquia de ações e objetivos (FREUND; BALTES, 2001b). A estratégia apareceu durante as entrevistas quando os atletas, percebendo perdas físicas relativas à saúde ocorridas a partir do estilo de vida que possuíam, decidiram eleger um comportamento novo que integrou uma dieta balanceada associada ao esporte:

\footnotetext{
"Eu quando comecei a correr, eu tinha 36 anos, eu era obeso, pesava 116 quilos e eu era alcoólatra e eu tinha uma opção de vida: o médico disse assim, tu não consegues chegar aos 40 anos se tu não parar, então o que eu comecei a fazer, eu comecei a fazer um regime, pois eu tinha que fazer uma dieta, porque eu não praticava esporte nenhum e o próprio alcoolismo não deixava praticar isso, né... então eu comecei a praticar por causa disso para emagrecer e tentar escapar do alcoolismo (A2 - 60 anos)".
}

Utilizando os recursos disponíveis: externos e internos, os atletas foram se adaptando a um novo padrão de vida que corresponde a um novo estilo de vida (FREUND; BALTES, 1998). De acordo com Sesc (2007), o conceito de estilo de vida é definido como o conjunto de ações habituais que refletem as atitudes, os valores e as oportunidades individuais. O conceito abarca pelo menos cinco fatores: a atividade física, os relacionamentos, a alimentação, o comportamento preventivo e o controle do estresse, no qual apresenta importância quando o que se quer é aumentar a expectativa de vida das pessoas. O estilo de vida é o fator que possui maior impacto no aumento da longevidade (SESC, 2007).

Durante os treinamentos a estratégia continuou aparecendo. Perdas de caráter físico percebidas estimularam os atletas a redefinir sua estratégia de treinamento, diminuindo a frequência e a intensidade dos exercícios:

"Eu me mantenho na atividade diminuindo a intensidade conforme as minhas condições físicas (A6 - 58 anos)".

Outra forma de perda percebida foram as lesões. Na situação de lesão os atletas direcionam sua energia no objetivo mais importante para o momento que é a cura da lesão (ROHR; LANG, 2009; FREUND; BALTES, 1998). Com isso, relatam diferentes formas de selecionar as ações. Os atletas 2 e 10 modificaram temporariamente a sua atividade física enquanto se recuperam: 
"[...] essa semana [...] eu comecei um trabalho de bicicleta ergométrica, eu não posso ficar parado [...] E agora eu tô fazendo bicicleta por causa disso, porque eu quero ver se eu cuido dessa lesão (A2 - 60 anos)".

"Fico lendo, gosto muito de ler, leio. Se não me impede de caminhar, continuo caminhando, claro. E se eu vejo que não dá para correr, eu caminho ao invés de correr (A10 - 74 anos)".

Outros atletas, porém, interrompem a atividade esportiva, ou reduzem a intensidade para tratar a lesão, com alguns realizando tratamento médico e fisioterápico:

"Paro, tomo remédios e faço fisioterapia (A3 - 45 anos)".

"Pára e cura primeiro, nada de tomar remédio. Claro, o atleta de ponta, estrela, tem todo esse conforto e até muita coisa, toma isso, aquilo, agente não, descansa (A7 - 75 anos)".

Como o corpo do atleta máster parece se adaptar mais lentamente ao treinamento, para conseguir obter um ótimo desempenho atlético, deve-se tentar criar um equilíbrio entre o estímulo que promova a efetividade do treinamento (fitness) e a fadiga. O maior desafio para o atleta máster é a manutenção de um nível de treinamento adequado que promova estímulo para o corpo e, ao mesmo tempo, a performance desejada, enquanto previne a fadiga, a frequência de lesões e o overtraining (MAHARAM et al., 1999). Com a ausência de estudos que determinem a dose-resposta de treinamento mais adequado para promover o fitness dos atletas master, nos diferentes esportes, assim como na corrida (SEGAL; CRESPO; SMIT, 1998), cabe aos próprios atletas, conforme os relatos, experimentarem o que mais se adapte a eles selecionando a melhor maneira de treinar, a partir de suas perdas.

Na perspectiva life-span, as lesões podem ser caracterizadas como um evento não-normativo, em função de que as pessoas, independente da idade que possuem, podem ser acometidas por lesões, sendo esportistas ou não (SUGARMAN, 2001; NERI, 2002). Mas para os atletas, as lesões são como companhias frequentes cujos fatores desencadeantes podem ser variados. No caso dos atletas entrevistados, eles apresentam a peculiaridade de estarem em um momento de vida no qual muitos estão iniciando, assim como outros experimentam há algum tempo, as alterações físicas próprias ao processo de envelhecimento (BIEUZEN et al., 2010; BEST; HART, 2008; POWELL, 2005; HAWKINS, WISWELL; MARCELL, 2003). Embora não se saiba com exatidão se a idade possa ser um fator que aumente as chances dos atletas desenvol- 
verem lesões, há outros fatores que contribuem para o aumento da frequência, tais como uma ingesta calórica reduzida, excesso de treinamento (SBME, 2003), ações mal executadas, acidentes e/ou fator psicológico (BECKER, 2000).

Ao selecionar as ações no esporte, seja de forma eletiva ou baseada em perdas, os atletas restringiram seu envolvimento em um número grande de objetivos, elegendo aqueles que foram mais pertinentes para sua vida em determinado momento. Com isso, eles conseguiram se adaptar aos diferentes desafios que a vida lhes impôs promovendo desenvolvimento pessoal e no esporte.

Além de conhecer de que forma os atletas se adaptam as perdas durante o desenvolvimento, é importante destacar que, em âmbito geral, o artigo também discutiu a maneira que essas pessoas estão construindo, ou vivenciando o seu processo de envelhecimento.

Envelhecer praticando esportes, ou iniciar a prática de um esporte somente na velhice é apenas uma das maneiras que as pessoas encontram para continuarem ativos e demonstrarem que traçam objetivos pessoais. Cada vez mais podemos observar idosos ou pessoas que estão iniciando o período de vida da terceira idade buscarem alternativas ou atividades que proporcionem prazer e bem-estar de acordo com suas preferências e anseios. Com base nos resultados ou nas informações coletadas, foi possível vislumbrar um conceito importante da gerontologia, ou seja, que é preciso envelhecer de forma bem-sucedida.

O conceito de Envelhecimentro Bem-sucedido é abrangente e resulta de décadas de discussões com poucas conclusões a respeito do termo. No artigo proposto focou-se o Envelhecimento bem-sucedido na perspectiva da psicologia do envelhecimento e tentou-se vislumbrar a partir do modelo proposto por Baltes e Baltes (1990) como atletas estão construindo seu envelhecimento de forma satisfatória e digna.

\section{Considerações Finais}

Considera-se que as estratégias de gerenciamento de vida foram fundamentais para estes esportistas, pois com o seu uso conseguem continuar na prática esportiva, apesar das dificuldades encontradas e dos declínios físicos sentidos durante os anos. Os atletas que participaram do estudo possuem a corrida como um hábito instituído em suas vidas. A vida de cada atleta é organizada em função do esporte e a partir disso se observa que possuem uma alta perícia adaptativa, pois as dificuldades vivenciadas não os fazem desistir de praticar o esporte. O gosto e o entusiasmo pelo esporte mostraramse como a principal motivação desses sujeitos. 
Perdas físicas obtidas por hábitos alimentares prejudiciais e vícios fizeram com que alguns dos entrevistados procurassem o esporte como uma forma de recuperar a saúde. Da mesma forma, perdas físicas percebidas durante anos de prática esportiva, ou as próprias lesões entendidas como perdas pelos atletas mudaram a forma de agir, ou pensar o esporte para continuarem por mais tempo na prática e assim obter ganhos apesar das intempéries.

Como adaptações que encontramos neste estudo estão atitudes preparatórias para o momento da competição, excluindo ações que diminuem a performance. Também foi indicada, pelos atletas, a diminuição da intensidade e da frequência dos exercícios quando perceberam que suas condições físicas estão declinando e uma troca de direção do foco do atleta para o autocuidado no advento de lesões. Todas essas foram medidas seletivas adotadas pelos atletas.

O presente artigo discutiu apenas uma fração do modelo SOC, ou seja, a estratégia da seleção que se subdivide - seleção eletiva ou seleção baseada em perdas. A pesquisa completa apresentou as quatro estratégias do modelo adicionadas a outras questões que nortearam o estudo e que contribuíram para sua conclusão. Dessa forma, contribuímos com a temática a partir do enfoque dado e sugerimos que outros pesquisadores discutam o mesmo tema de outras formas ou com outras abordagens teóricas. Sugere-se também que o número de sujeitos participantes do estudo seja aumentado.

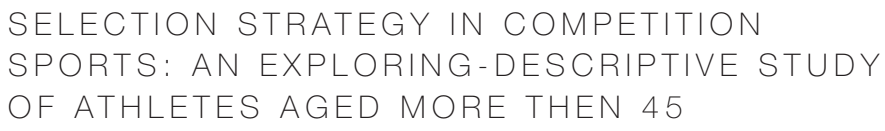

Baltes \& Baltes (1990) proposed an adapting developing model called selection, optimization and compensation (SOC Model). This essay analyzed the selection strategy, and focused on the study of how running athletes selected their actions in sports. A first screening test was performed with 83 athletes, and 11 of them (average age 59,91 $\pm 0,58$ ) answered questions and were submitted to a semi-structured interview. Data from the interviews showed that athletes used selection strategy in order to direct their lives or to overcome any loss/difficulties, and thus they kept their practicing or even improved their sports performance for a longer period. 
ALLISON, Devan; SEALS, Douglas. Vascular Health in the Ageing Athlete. Experimental Physiology. v. 97, n. 3, p. 305-310, mar. 2012.

American College of Sports Medicine (ACSM). American College of Sports Medicine Position Statement on: the use of alcohol in sports. 2010. 6p. Disponivel em <http:// unw.medscape.com/viewarticle/716247> Acesso em: 11 fev. 2012.

BAJOR, Janice; BALTES, Paul. The relationship between Selection Optimization with Compensation, conscientiousness, motivation, and performance. Joumal of Vocation Behavior, v. 63, p. 347-367, dec. 2003.

BALTES, Paul; BALTES, Margret. Psychological Perspectives on Successful Aging: The Model of selective optimization with Compensation. In: Successful Aging: Perspectives from the behavioral Sciences. New York: Cambridge University Press, 1990, p. 1-34.

BALTES, Paul; CARSTENSEN, Laura. The Process of Successful Aging. In: MARKSON, Elizabeth; HOLLIS, Lisa. Intersections Of Aging - Readings in Social Gerontology. Los Angeles: Roxbury Publishing Company, 2000, p. 65-81.

BALTES, Paul; Theoretical Propositions of Life-Span Developmental Psychology: On the Dynamics Between Growth and Decline. Developmental Psychology, v. 23, n. 5, p. 611-626, sept.1987.

BALTES, Paul; STAUDINGER, Ursula; LINDENBERGER, Ulman. Lifespan Psycology: theory and application to intellectual functioning. Annual Reviews Psychology. v. 50, p. 471-507, jan. 1999.

BECKER, Beno. Psicologia Aplicada às Lesões. In: BECKER, Beno. Manual de Psicologia do Esporte e Exercício. Porto Alegre: Nova Prova, 2000. p. 283-295.

BENTO, Jorge. O Século do Idoso e o Papel do Desporto. Terceira Idade, Brasília, n. 46, p.15-23, out. 1999.

BEST, Thomas; HART, Lawrence. A Growing Concern. Clinical Journal of Sports Medicine, v. 18, n. 6, p. 477-478, nov. 2008.

BIEUZEN, François et al., Age-Related Changes in Neuromuscular Function and Performance following a High-Intensity Intermittent Task in Endurance-Trained Men. Gerontology, v. 56, p. 66-72, jan. 2010.

BIRREN, James. A Brief History of the Psychology of Aging. The Gerontologist, n. 1, p. 69-77, June 1961.

BIRREN, James; SCHROOTS, J. The History of Geropsychology. In: BIRREN, James; SCHAIE, Warner. Handbook of the Psychology of Aging. Califórnia: Academic Press, 2001. p. 3-28.

BROWN, Martha. Special Considerations During Rehabilitation of Aged Athlete. Clinics in Sports Medicine. v. 8, n. 4, p. 893-901, Oct. 1989.

CAMPBELL, Wayne; GEIK, Rachel. Nutritional considerations for the Older Athlete. Nutrition. v. 20, p. 603-608, June 2004

CHEN, Andrew; MEARS, Simon; HAWKINGS, Richard. Orthopaedic Care of the Aging Athlete. Journal of the American Academy of Orthopaedic Surgeons. v. 13, n. 6, p. 407416, apr. 2005.

FAULKNER, John et al. The Aging of Elite Male Athletes: age-related changes in performance and skeletal muscle structure and function. Clinical Joumal of Sports Medicine. v. 18, n. 6, p. 501-507, nov. 2008. 
FREUND, Alexandra; BALTES, Paul. Selection, Optimization, and Compensation as Strategies of Life Management: Correlations with Subjective Indicators of Successful Aging. Psycology and Aging. v. 13, n. 4, p. 531-543, 1998.

FREUND, Alexandra; Selection, Optimization, and Compensation as Strategies of Life Management: Correction to Freund and Baltes (1998). Psycology and Aging. v. 14, n. 4 , p. 700-702, 1999.

FREUND, Alexandra; The adaptiveness of Selection, Optimization, and Compensation as Strategies of Life Management: Evidence From a Preference Study on Proverbs. Joumal of Gerontology. Psychologial Sciences. v. 57b, n. 5, p. 426-434, 2002.

GASKELL, George. Entrevistas Individuais e Grupais. In: BAUER, M.; GASKELL, G. Pesquisa Qualitativa com Texto, Imagem e Som. Um Manual Prático. Rio de Janeiro: Vozes, 2003. p. 64-89.

GIL, Antônio. Métodos e Técnicas de Pesquisa Social. São Paulo: Atlas, 1987.

GOLDIM, José. Manual de Iniciação à Pesquisa em Saúde. Porto Alegre: Da Casa Editora, 1995.

HAWKINS, Steven; WISWELL, Robert; MARCELL, Taylor. Exercise and the Master Athlete - A Model of Sucessful Aging? Journal of Gerontology: Medical Sciences, v. 58a, n. 1, p. 1009-1011, nov. 2003.

JOPP, Daniela; SMITH, Jacqui. Resources and Life-Management Strategies as Determinants of Successful Aging: On the Protective Effect of Selection, Optimization, and Compensation. Psychology and Aging. v. 21, n. 2, p. 253-265, June. 2006.

LI, Shu-Chen; FREUND, Alexandra. Advances in Lifespan Psychology: A Focus on Biocultural and Personal Influences. Research in Human Development, v. 2 n. 1 e 2, p. 1-23, june. 2005.

LICHTENFELS, Henriete. O Envelhecimento Humano na periferia: um diálogo entre idosos moradores da periferia e a perspectiva da psicologia do desenvolvimento do curso de vida, life-span. São Leopoldo: Instituto Ecumênico de Pós-Graduação em Teologia, 2007. 272p. Tese, Doutorado em Teologia, São Leopoldo, 2007

MAHARAM, Lewis et al. Master Athletes - Factors Affecting Performance. Sports Medicine. v. 28, n. 4, p. 273-274, oct.1999.

MINAYO, Maria Cecília. O Desafio do Conhecimento: pesquisa qualitativa em saúde. São Paulo: Hucitec-abrasco, 1992.

MORLEY, John. The Aging Athlete. Journal of Gerontology: Medical Sciences, v. 55a, n. 11, p. $627-629,2000$

NERI, Anita. Paradigmas Contemporâneos sobre o Desenvolvimento Humano em Psicologia e em Sociologia. In: NERI, Anita. (Org.) Desenvolvimento e Envelhecimento. Perspectivas biológicas, psicológicas e sociológicas. São Paulo: Papirus, 2001. p. 11-37.

NERI, Anita. Teorias Psicológicas do Envelhecimento. In: FREITAS, E.; PY, L.; NERI, A. (Orgs.) Tratado de Geriatria e Gerontologia. Rio de Janeiro: Guanabara-Koogan, 2002. p. 32-46.

POWELL, Amy. Issues Unique to the Masters Athlete. Current Sports Medicine Reports. v. 4, n. 6, p. 335-340, dec. 2005

RICHARDSON, Roberto. Pesquisa Social. Métodos e Técnicas. 3 ed. São Paulo: Atlas, 1999

ROHR, Margund; LANG, Frieder. Aging Well Together - A Mini-Review. Gerontology. v. 55, p. 333-343, 2009. 
SANTOS, Marinella; TINUCCI, Taís. O Consumo de Álcool e o Esporte: uma visão geral em atletas universitários. Revista Mackenzie de Educação Física e Esporte. v. 3, n. 3, p. $27-43,2004$

STAUDINGER, Ursula M.; MARSISKE, Michael; BALTES, Paul. Resiliência e níveis de capacidade de reserva na velhice: perspectivas da teoria do curso de vida. In: NERI, Anita Liberalesso. (Org.) Psicologia do envelhecimento. Campinas: Papirus, 1995. p. 195-228.

SBME - Sociedade Brasileira de Medicina do Esporte. Diretriz da Sociedade Brasileira de Medicina do Esporte. Modificações dietéticas, reposição hídrica, suplementos alimentares e drogas: comprovação de ação ergogênica e potenciais riscos a saúde. Revista Brasileira de Medicina do Esporte. v. 9, n. 2, p. 43-56, 2003.

SCHROOTS, Johannes. Theoretical Developments in the Psychology of Aging. The Gerontologist, Washington, v. 36, n. 6, p. 742-748, 1996

SEGAL, Doralie; CRESPO, Carlos.; SMIT, Ellen. Active Seniors. Protect Them, Don't Neglect Them. Public Health Reports. v. 113, p. 137-139, mar./apr. 1998.

SESC. Lazer Ativo: o que é? Disponível em: <http://wmw.sesi.org.br/portal/main.jsp>. Acesso em: 9 dez. 2007

SUGARMAN, Léonie. Life-span Development. Frameworks, Accounts and Strategies. Canada \& USA: Psychology Press Ltda, 2001.

YEUNG, Danni; FUNG, Helene. Aging and Work: How Do SOC Strategies Contribute to Job Performance Across Adulthood? Psychology and Aging, v. 24, n. 4, p. 927-940, dec. 2009. 